\title{
Central Odontogenic Fibroma - A Pediatric Variant
}

\author{
Pavan $\mathrm{P}^{1}$, Shyam Prasad Reddy D2 , Pavan G Kulkarni ${ }^{3}$
}

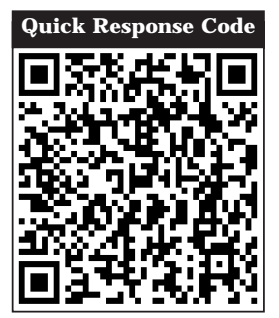

doi : $10.5866 / 2014.621559$

1Post-Graduate Student

2Senior Lecturer

${ }^{3}$ Reader

Department of Oral \& Maxillofacial Pathology

Kamineni Institute of Dental Sciences,

Narketpally, Nalgonda (Dist), Telangana, India

\section{Article Info:}

Received: J anuary 12, 2014

Review Completed: February 11, 2014

Accepted: March 11, 2014

Available Online: J uly, 2014 (www.nacd.in)

(c) NAD, 2014 - All rights reserved

Email for correspondence:

shyamprasadreddy_d@yahoo.co.in

\begin{abstract}
:
Central odontogenicfibroma (CODF) is an extremely rare benign tumor accounting for $0.1 \%$ of all odontogenic tumors. CODF is defined as a fibroblastic neoplasm containing varying amounts of apparently inactive odontogenic epithelium. It is considered to be derived from mesenchymal tissue of dental origin, periodontal ligament, dental papilla, or dental follicle. The peak incidence of CODFs has been observed in the second decade of life and shows a female predilection. The World Health Organization (WHO) recognizes two variants of CODF namely epithelial rich type (WHO) and epithelial poor type (simpletype). TheWHO type contains mineralized material that is interpreted as osteoid, cementoid like or dysplastic dentin. The simple type of CODF is composed by a delicate fibrous connective tissue with considerable ground substance yielding a fibromyxoid quality. Here we report a case of simple type of CODF in a 13 year old boy who presented with pain and swelling in the anterior region of the mandible.
\end{abstract}

Key words: Central odontogenic fibroma, mandible, pediatric.

\section{Introduction:}

Odontogenic fibroma is classified as a mesenchymal odontogenic tumor. ${ }^{1}$ Odontogenic fibroma can be further divided into central (intraosseous) odontogenic fibroma (CODF) and peripheral (extraosseous) odontogenic fibroma (PODF) according to the anatomical sites involved. ${ }^{2}$ Central odontogenic fibroma (CODF) is an extremely rare benign tumor accounting for only $0.1 \%$ of all odontogenic tumors. ${ }^{3}$ The lesion may evolve from a dental germ (dental papilla or follicle) or from the periodontal membrane, and therefore is invariably be related to the coronal or radicular portion of teeth. ${ }^{4}$ It occurs most often in females, usually located in the anterior aspect of the maxilla, presenting as a well circumscribed radiolucency that may induce root resorption. ${ }^{3}$ 
Clinically, CODF presents as a slow growth asymptomatic mass which, in most cases, can remain unknown until the appearance of a swelling. In more severe cases root resorption and displacement of adjacent teeth have been reported. ${ }^{5}$ The CODF consists of collagenous fibrous connective tissue containing varying amounts of odontogenic epithelium. ${ }^{6}$

In 1980, Gardner described odontogenic fibroma and classified it into three different related lesions namely (1) the hyperplastic dental follicle, (2) a fibrous neoplasm with varying collagenous fibrous connective containing nests of odontogenic epithelium (CODF simple type) and (3) a more complicated lesion with features of dysplastic dentin or cementum like tissue with varying amounts of odontogenic epithelium (WHO type) which was later designated as CODF WHO type. ${ }^{7}$ Here we describe a case of CODF (simple type) in a 13 year old boy occurring in the anterior mandibular region.

\section{Case report}

A 13 year old male reported with the complaint of pain and swelling in the left lower front tooth region since 2 months. On extraoral examination, a diffuse swelling was seen in the mandibular anterior region extending from midline to $2 \mathrm{~cm}$ anterior to ear lobule on left side producing facial asymmetry. The swelling was hard in consistency, tender on palpation with local rise of temperature. The skin over the swelling was normal and regional submandibular Iymphnodes were tender on palpation. Medical, surgical and family histories were not noteworthy.

On intra oral examination, a solitary swelling measuring about $4 \times 4 \mathrm{~cm}$ was seen extending from mesial aspect of 33 to distal aspect of 35, obliterating lower vestibule (Figure 1). Expansion of buccal cortical plate and displacement of teeth (31, 33, 34, 35 and 41) were also seen. The swelling was hard in consistency, tender on pal pation al ong with mobility of teeth - 31, 34 and 35. Patient was advised for routine hematological and radiographic investigations. An OPG was advised which showed unilocular radiolucency extending from left mandibular first molar to right mandibular first premolar i.e., 36 to 44 , al ong with root resorption of 31,32 and 41 and; an impacted right mandibular canine (Figure 2). Occlusal radiograph revealed buccolingual cortical expansion (Figure 3). From the above clinical and radilogical findings a provisional diagnosis of unicystic ameloblastoma was made.

An incisional biopsy was performed and sent for histopathological examination. Sections were stained with routine $\mathrm{H} \& \mathrm{E}$. Microscopic examination showed highly cellular fibrous connective tissue stroma with plump fibroblasts and numerous scattered inactive appearing odontogenic epithelium islands. Many hemorrhagic areas and moderate to severe inflammatory cell infiltrate was also evident (Figure 4 and 5). The above histological features along with clinical and radiographic findings were suggestive of simple type of central odontogenic fibroma.

\section{Discussion:}

The central odontogenic fibroma (CODF) remains incompletely understood. ${ }^{8}$ CODF is defined as a fibroblastic neoplasm containing varying amounts of apparently inactive odontogenic epithelium. ${ }^{9}$ According to Covani et al the CODF was believed to originate from mesenchymal odontogenic tissue such as dental papilla, periodontal ligament, or dental follicle. Considering the histogenesis of the lesion in the WHO classification, it has been suggested that the epithelium poor type of CODF is derived from the dental follicle whereas the epithelium rich type arises from periodontal ligament. ${ }^{10}$ The literature review showed that CODF is a very rareodontogenic neoplasm accounting for $0.1 \%$ of odontogenic tumors. ${ }^{3}$ It is reported in wide age groups and frequently diagnosed in patients between the second and fourth decades of life and in the present case it occurred in the second decade. CODF mostly show female predilection with a ratio of 2.2:1.1 ${ }^{11}$ Both the jaws have been affected equally. In the maxillary arch, it involves anterior segment and mandibular lesions affect the premolar and molar areas. ${ }^{9}$ Most of the times in maxilla CODF occurs in anterior region accounting for $29 \%$, whereas premolar and molar region accounts for $6 \%$ and $10 \%$ respectively. 


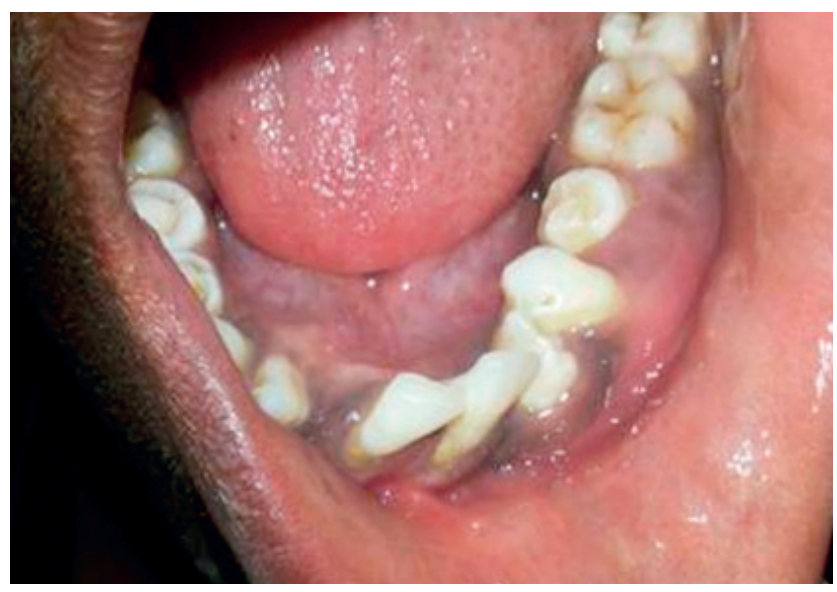

Figure 1: Clinical photograph showing a solitary swelling extending from mesial aspect of left mandibular canine to distal aspect of left mandibular second premolar obliterating lower vestibule along with buccolingual expansion of the cortical plates.

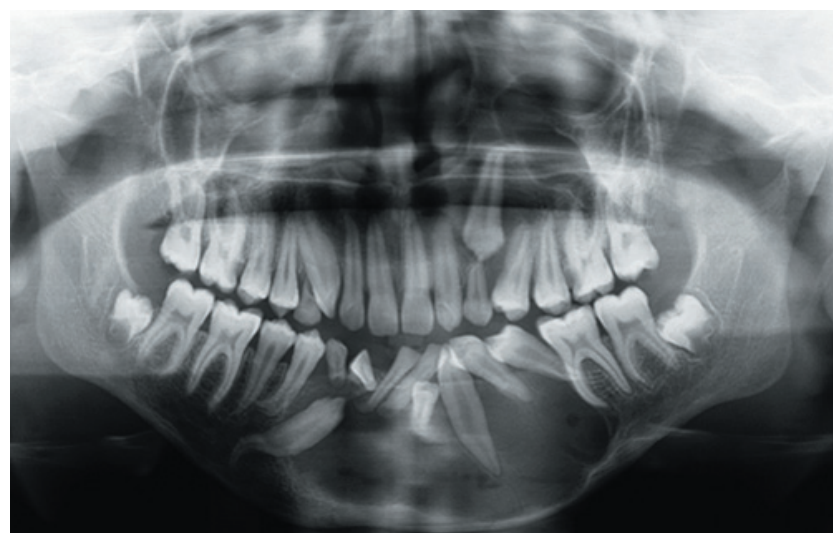

Figure 2: OPG showing unilocular radiolucency extending from left mandibular first molar to right mandibular first premolar i.e., 36 to 44, along with root resorption of 31, 32 and 41 and; an impacted right mandibular canine.

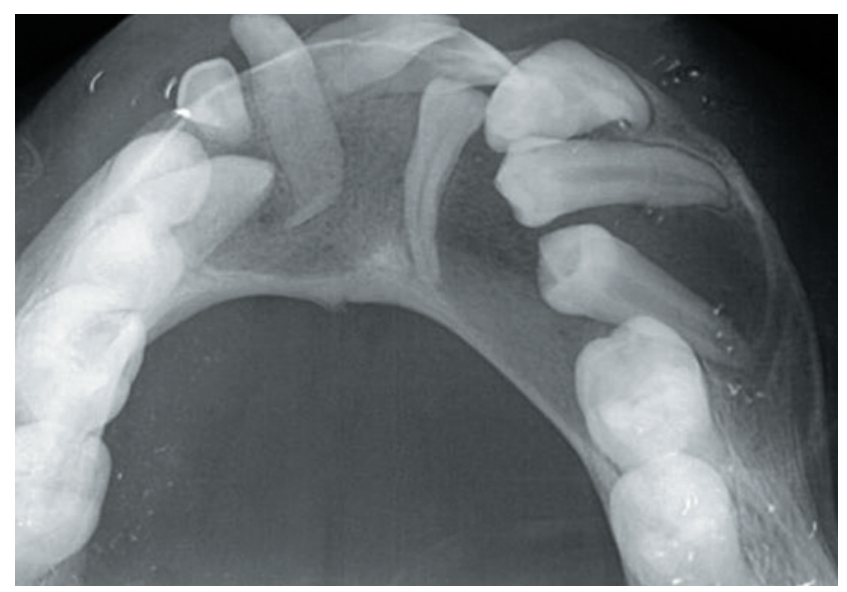

Figure 3: Occlusal radiograph showing buccolingual cortical expansion.

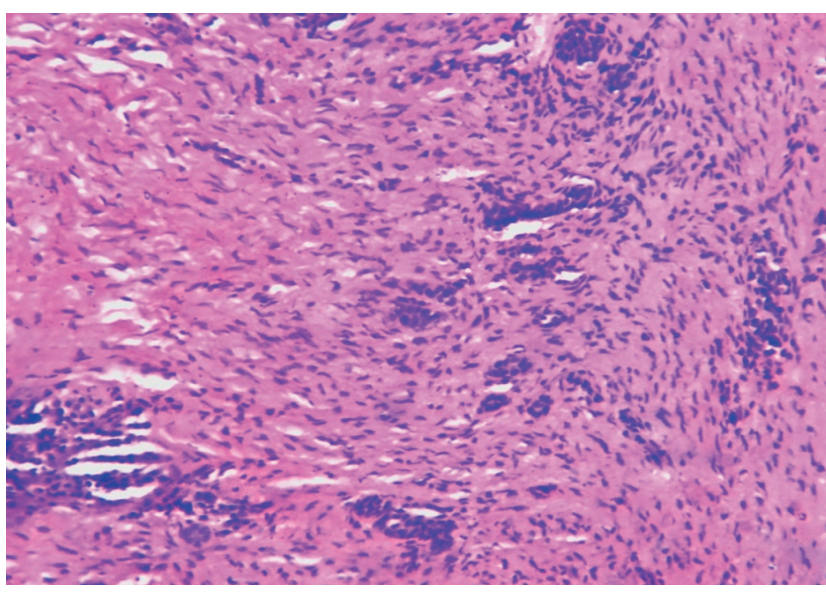

Figure 4: $\mathrm{H} \& \mathrm{E}$ stained section showing highly cellular fibrous connective tissue stroma with dense bundles of collagen fibres (10X).

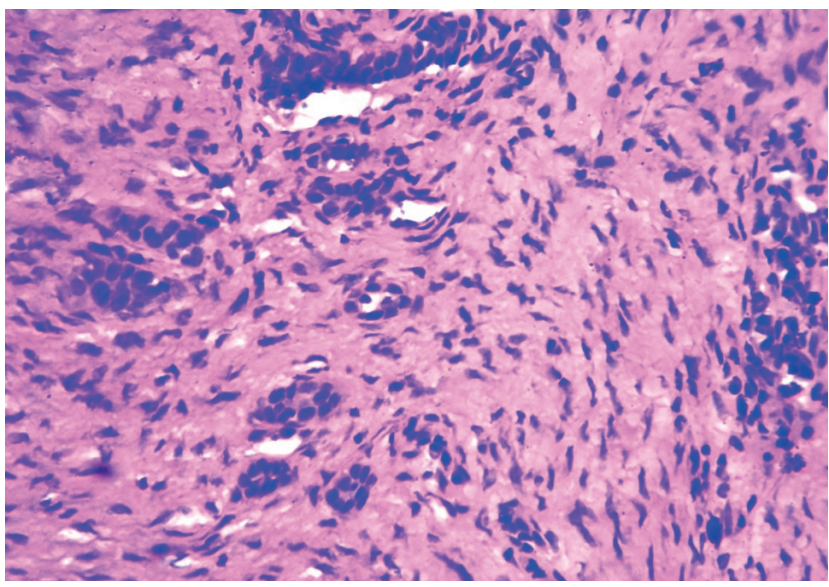

Figure 5: H \& E stained section showing highly cellular fibrous connective tissue stroma with plump fibroblasts and numerous scattered inactive appearing odontogenic epithelium islands (40X).

In mandible CODF occurs more commonly in molar region accounting for $29 \%$ whereas anterior region and premolar area accounts for $8 \%$ and $18 \%$ respectively. In the present case mandibular anterior region was involved in a male patient.

Most lesions are seen as painless swelling and appear as asymptomatic expansion of buccal and lingual cortical plates. It is seen as a slowly enlarging hard swelling which is sometimes recognized by the presence of slow growing diastema due to dislocation of adjacent teeth. ${ }^{12}$ In the present case the swelling was associated with pain and also there was displacement of teeth particularly 31, 33, 34, 35 and 41. 
Radiographically, the lesions are associated with the crown of an unerupted molar, premolar, or incisor tooth. CODF usually appears as a unilocular radiolucency with well defined borders, but may also exhibit a multilocular appearance with scalloped margins. ${ }^{12,13}$ Most cases of CODF are radiolucent (84.8\%), unilocular (56.4\%) with well defined borders (65.4\%). ${ }^{10}$ In the present case unilocular radiolucency was seen extending from left mandibular first molar to right mandibular first premolar i.e., 36 to 44, along with an impacted right mandibular canine.

Central odontogenic fibroma has been classically divided into two histological varients namely the Simpe type and the WHO type. ${ }^{8}$ The basic difference between simple and WHO type is that the stroma of the simple fibroma mimics that of a dental follicle from which it is probably derived. Histologically, the simple type exhibits relatively acellular delicatefibers which are interspersed with considerable amount of ground substance. On the other hand the stroma of the WHO type exhibits high cellularity. It occurs as fibroblastic strands which may be interwoven with less cellular areas. The epithelial rests are dispersed sparsely in the simpletype. In contrast, the odontogenic epithelium is an integral component of the WHO type. The other difference between these two variants is the presence of foci of calcifications of the collagenous materials in the WHO type which are described as cementoid, osteoid, and dysplastic dentin by several authors. ${ }^{9}$ In the present case highly cellular fibrous connective tissue stroma with plump fibroblasts and numerous scattered inactive appearing odontogenic epithelium islands were seen suggesting simple type of central odontogenic fibroma.

The mode of treatment for CODF is enucleation and curettage. Inspite of central odontogenic fibroma be usually easily removed, not showing any adherence to bone and/or tooth structure, the recurrences were related to insufficient curettage. Herein, because of their benign slow growth characteristic, a clinical identification of recurrence or residual disease could be identified only several years after. ${ }^{14}$

\section{Conclusion:}

Central odontogenic fibromas are rare lesions and most of them are confined to maxillary anterior region. This case report is an additional report for CODF occurring in anterior mandible in a 13 year old boy.

\section{References:}

1. Lewis R. Eversole. Odontogenic fibroma including amyloid and ossifying variants. Head Neck Pathol 2011; 5:335-343

2. Kyung-Soo Nah. Central odontogenic fibroma: a case report. Imag Sci Dent 2011; 41: 85-88

3. Daniels J . Central odontogenic fibroma of mandible: a case report and review of the literature. Oral Surg Oral Med Oral Pathol Oral Radiol Endod 2004; 98:295-300.

4. Marco $\mathrm{T}$ Brazao-Silva, Alexandre $V$ Fernandes, Antonio $\mathrm{F}$ Durighetto-J unior, Sergio V Cardoso, Adriano M Loyola. Central odontogenic fibroma - a case report with Iongterm followup. Head Face Med 2010; 6:20

5. Cawson R, Binnie W, Speight P, Barrett A, Wright J . Luca'spathology of tumors of the oral tissues. 5th ed. London: Churchill Livingstone; 1998. pg 71.

6. Ugo Covani, Roberto Crespi, Nicola Perrini, Antonio Barone. Central odontogenic fibroma: A case report. Med Oral Patol Oral Cir Bucal 2005; 10(2):154-157.

7. Gardner DG. The central odontogenic fibroma: an attempt at clarification. Oral Surg Oral Med Oral Pathol 1980; 425432.

8. Gardner DG. Central odontogenic fibroma current concept. J Oral Pathol Med 1996; 25:556-561.

9. V Veeravarmal, R Nirmal Madhavan, M Mohamed Nassar, R Amsaveni. Central odontogenic fibroma of the maxilla. J OMFP 2013; 17.

10. Felipe Rodrigues de Matos, Maiara de Moraes, Antonio Capistrano Neto, Marcia da Costa Miguel, Ericka J anine da Silveria. Central odontogenic fibroma. Annals Diag Path 2011; 15:481-484

11. Neville BW Damm DD. Oral and maxillofacial pathology, 2nd edition.

12. Kaffe I, Buchner A. Radiological features of central odontogenic fibroma. Oral Surg Oral Med Oral Pathol 1994; 78: $811-818$

13. Adal berto MT, Guillermo MM, Roman CB, Pablo AV, Victor TR,Ana María CV. Central odontogenic fibroma: New findings and report of a multicentric collaborative study. Oral Surg Oral Med Oral Pathol Oral Radiol Endod 2011; 112:349-358.

14. Heimdal A, Isacsson G, Nilsson L: Recurrent central odontogenic fibroma. Oral Surg Oral Med Oral Pathol 1980; 50(2):140-145 